\title{
Intrinsic instability of electronic interfaces with strong Rashba coupling
}

\author{
S. Caprara ${ }^{1,2}$, F. Peronaci ${ }^{1}$, and M. Grilli ${ }^{1,2}$ \\ ${ }^{1}$ Dipartimento di Fisica, Università di Roma "La Sapienza", P. le Aldo Moro 5, 00185 Roma, Italy and \\ ${ }^{2}$ ISC-CNR and Consorzio Nazionale Interuniversitario per le Scienze Fisiche della Materia, Unità di Roma "Sapienza"
}

(Dated: June 1, 2022)

\begin{abstract}
We consider a model for the two-dimensional electron gas formed at the interface of oxide heterostructures, which includes a Rashba spin-orbit coupling proportional to the electric field perpendicular to the interface. Based on the standard mechanism of polarity catastrophe, we assume that the electric field has a contribution proportional to the electron density. Under these simple and general assumptions, we show that a phase separation instability (signaled by a negative compressibility) occurs for realistic values of the spin-orbit coupling and of the electronic band-structure parameters. This provides an intrinsic mechanism for the inhomogeneous phases observed at the $\mathrm{LaAlO}_{3} / \mathrm{SrTiO}_{3}$ or $\mathrm{LaTiO}_{3} / \mathrm{SrTiO}_{3}$ interfaces.

PACS numbers: 71.70.Ej,73.20.-r,73.43.Nq,74.81.-g
\end{abstract}

The observation of a two-dimensional (2D) metallic state at the heterointerface of two insulating oxides $\left[\mathrm{LaAlO}_{3} / \mathrm{SrTiO}_{3}(\mathrm{LAO} / \mathrm{STO})\right]$ [1] remarkably discovered a new class of high-mobility electron gases (EGs) important both for fundamental and applicative reasons. The occurrence of superconductivity in this 2DEG [2, 3] and in $\mathrm{LaTiO}_{3} / \mathrm{SrTiO}_{3}(\mathrm{LTO} / \mathrm{STO})$ [4, 5], with the possibility to tune the charge density by gating, has further attracted great attention. On the other hand, there is increasing evidence that electron inhomogeneity plays a relevant role in these systems. Not only the large width of the superconducting transition in transport experiments is a clear indication of charge inhomogeneity [6, 7], but also magnetometry and tunnelling experiments $[8-$ 11] find submicrometric inhomogeneities. While impurities, defects, and other extrinsic mechanisms (see, e.g. Ref. [12]) surely induce inhomogeneities, the recent discovery of negative compressibility in a low filling regime [13] is a stringent demonstration that an intrinsic mechanism (like, e.g., an effective attraction) is at work to render these 2DEGs inhomogeneous by charge segregation and phase separation even in a perfectly clean and homogeneous system. Moreover, even if such mechanism were not strong enough to drive the system unstable, it would increase the charge susceptibility, emphasizing the effects of the extrinsic mechanisms (impurities, defects and so on).

In this Letter, we point out that a generic source of phase separation is provided by the Rashba spin-orbit coupling (RSOC), whenever the electric field determining this coupling also controls the electron density. This is precisely the case of LAO/STO and LTO/STO (generically, LXO/STO) interfaces, where the two following conditions remarkably meet: i) a strong electric field occurs perpendicular to the interface because of the polarity catastrophe [14 16] and brings the electrons at the oxide interface to produce the 2DEG. An additional electric field, although quantitatively less important, is introduced by the gating potential and tunes the density of the $2 \mathrm{DEG}$; ii) the parity symmetry is broken at the interface naturally entailing a RSOC, which experiments have found to be substantial [17, 18]. We show that these two concomitant conditions are enough to drive the 2DEG unstable towards phase separation, thereby providing a general and intrinsic mechanism for the inhomogeneity of these oxide interfaces.

The mechanism for RSOC-induced phase separation is rather simple. In a metallic system with a rigid band structure the chemical potential $\mu$ increases upon increasing the electron density $n$ and the compressibility $\kappa \equiv \partial n / \partial \mu$ is positive. On the other hand, if the band structure is modified by the charge density (like, e.g., in strongly correlated systems, where the quasiparticle bandwidth increases moving away from the half-filled Mott-Hubbard insulator) the possibility may occur that $\mu$ decreases with increasing $n$ and $\kappa<0$, with the negative compressibility region signaling a phase separation. The mechanism at work is so generic that it could be relevant for many other systems in the presence of RSOC like, e.g., surface states of topological insulators, InAs or GaAs semiconductor heterostructures, or heavy metal surface alloys.

Our analysis finds that a band structure with large (even anisotropic) masses together with a rather isotropic Rashba coupling are favorable conditions for this instability to occur. We also stress that the charge inhomogeneity within the interface is due to an inhomogeneous electronic reconstruction and therefore it is balanced by a correspondingly inhomogeneous redistribution of the countercharges on the top layer. Therefore the inhomogeneity is not prevented by the standard Coulomb mechanisms leading to frustrated phase separation in other systems 19 21]. This naturally leads to large submicrometric inhomogeneities, like those detected in LXO/STO.

Fig. 1(a) schematically shows the 2DEG between the two oxide layers, the gating layers and the resulting electric fields. The electron reconstruction to avoid the polarity catastrophe is also depicted with the electron charge 


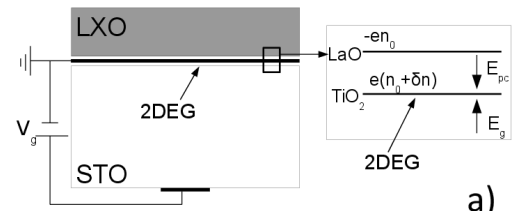

a)
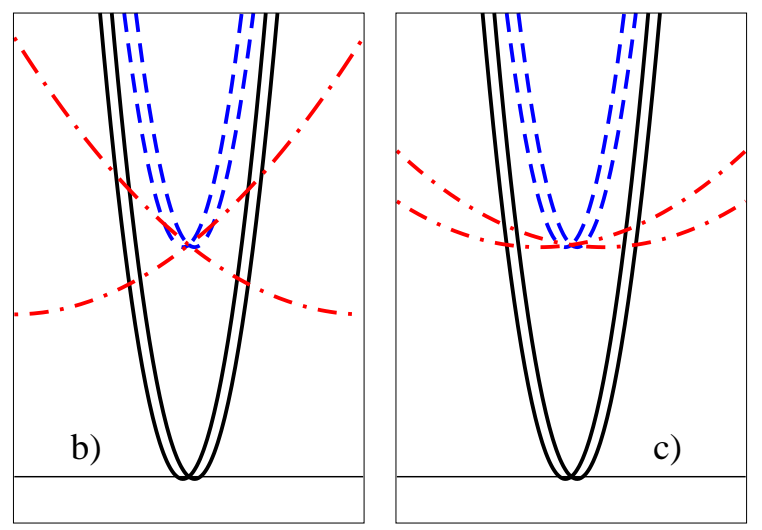

FIG. 1. (Color online) (a) Schematic view of the LXO/STO interface in the presence of gate potential $V_{g} . n_{0}$ is the density of the electrons transferred to the interface by the polarity catastrophe and giving rise the $E_{P C}$ electric field. $\delta n$ is the electron density tuned by the gating field $E_{g}$. (b) Schematic band structure for the $t_{2 g}$ bands along $k_{x}$ with $\nu=30$ and $\eta=1$ and (c) $\eta=1 / \nu$. We report the $d_{x y}$ (solid black), $d_{x z}$ (dashed blue online), $d_{y z}$ (dot-dashed, red online) bands.

density $n_{0}$ transferred onto the interface, leaving oppositely charged planes in the LXO layers above it. As a consequence, a field $E_{P C}=e n_{0} /\left(\epsilon_{0} \epsilon_{1}\right)$ arises perpendicular to the interface, where $\epsilon_{1} \sim 20$ is the LXO dielectric constant, $\epsilon_{0}$ is the vacuum dielectric permittivity and $e$ is the electron charge. Typical values of $E_{P C}$ may reach $10^{8} \mathrm{~V} / \mathrm{m}$. The total electric field perpendicular to the interface is obtained by adding to $E_{P C}$ the gate electric field $E_{g}=V_{g} / d$, where $d$ is the width of the STO substrate, which tunes the charge density with a term $e \delta n=\epsilon_{0} \epsilon_{2} E_{g}$. For a STO substrate $0.5 \times 10^{-3} \mathrm{~m}$ thick, $E_{g} \sim 10^{5} \mathrm{~V} / \mathrm{m}\left(\ll E_{P C}\right)$. It is quite important to notice that the large value of the low-temperature STO dielectric constant $\epsilon_{2} \gtrsim 10^{4}$ allows for relatively large variations of the electron filling $\delta n$, while the gate electric field always stays much smaller than $E_{P C}$. As a consequence, the 2DEG can be greatly depleted when $\delta n \sim-n_{0}$, while the total electric field is still large $E=E_{g}+E_{P C} \approx E_{P C}$.

For a description of the STO band structure, where the electrons of the LXO/STO interfaces mainly reside, we consider three parabolic bands schematizing the bottom of the $t_{2 g}$-like bands of Ti. The lowest $\left(d_{x y}\right)$ band has light isotropic mass $\sim 0.7 m_{0}\left(m_{0}\right.$ is the bare electron mass) while at an energy $\Delta \approx 50 \mathrm{meV}$ above there are two $\left(d_{x z}\right.$ and $\left.d_{y z}\right)$ bands with the same light mass in one direction and a heavy mass as large as $20 m_{0}$ in the other 15, 22 27]. For simplicity, we neglect all mixings among the three bands [28].

The lowest band has therefore isotropic dispersion

$$
\varepsilon_{ \pm}(k)=\frac{\hbar^{2} k^{2}}{2 m} \pm \alpha k
$$

which is the prototypical model for 2DEGs with Rashba coupling $\alpha$ (see, e.g., Ref. [30]) and gives rise to a 2D isotropic band structure composed of two branches [solid black curves in Fig. 1 (b),(c)] split by $\Delta_{k}=2 \alpha|k|$, with a minimum $-\varepsilon_{0} \equiv-m \alpha^{2} /\left(2 \hbar^{2}\right)$ occurring on a circle of radius $\alpha m$. The corresponding density of states (DOS) is $N(\varepsilon)=N_{0} \equiv m /\left(\pi \hbar^{2}\right)$ for $\varepsilon>0$ and $N(\varepsilon)=$ $N_{0} / \sqrt{1+\varepsilon / \varepsilon_{0}}$ for $-\varepsilon_{0}<\varepsilon \leq 0$. The other two bands give rise to (strongly) anisotropic dispersions

$$
\varepsilon_{ \pm}(k)=\frac{\hbar^{2} k_{x}^{2}}{2 m_{x}}+\frac{\hbar^{2} k_{y}^{2}}{2 m_{y}} \pm \sqrt{\alpha_{x}^{2} k_{x}^{2}+\alpha_{y}^{2} k_{y}^{2}}+\Delta,
$$

with $\nu \equiv m_{y} / m_{x} \sim 30$ and $\eta \equiv \alpha_{y} / \alpha_{x}$ for the $d_{x z}$ band and $\nu \equiv m_{x} / m_{y} \sim 30$ and $\eta \equiv \alpha_{x} / \alpha_{y}$ for the $d_{y z}$ band. The DOS arising from Eq. (2) depends on $(\varepsilon-\Delta) / \varepsilon_{0}$ only and is given in terms of complete elliptic integrals of the first and third kind (see Supplemental Material). The precise form of $\alpha_{x}$ and $\alpha_{y}$, and therefore of $\eta$, should be determined by first-principle calculations, which are beyond the scope of this Letter. However, we argue that $\eta$ should be intermediate between two extreme cases: $\eta=1$ (isotropic Rashba coupling) and $\eta=1 / \nu$ [i.e. $\alpha_{x, y}=$ $1 / m_{x, y}$ to reconstruct the relativistic form of the spinorbit coupling: $(\mathbf{v} \times \sigma) \cdot \hat{\mathbf{E}}]$. The band bottom occurs at $\Delta-\nu \varepsilon_{0}$ in the former case and at $\Delta-\varepsilon_{0}$ in the latter (see Fig. 1(b) and (c)).

In any case, $\alpha$ and $\alpha_{x, y}$ depend on the perpendicular electric field $E$ but, instead of the customary simple proportionality, we here take a non-linear expression $\alpha(E)=\tilde{\alpha} E /(1+\beta E)^{3}$ which is also valid at large fields and stems from a standard derivation of the Rashba coupling [30, 31] (see also, e.g., Eq. (2) in Ref. 32] and Supplemental Material of this Letter). Thus, $\varepsilon_{0}=$ $\gamma E^{2} /(1+\beta E)^{6}$ [with $\gamma \equiv m \tilde{\alpha}^{2} /\left(2 \hbar^{2}\right)$ ] is an increasing function of $E$ up to moderate-large values $[\sim 1 /(2 \beta)]$. The key point here is that the electric field is directly related to the number of electrons in the plane $E(n)=$ $E(0)+n E^{\prime}$ and therefore the larger is $n$, the deeper is the energy minimum $\varepsilon_{0} \sim\left[E(0)+n E^{\prime}\right]^{2}$. This may render energetically convenient for the system to attract electrons to have a deeper energy minimum, where more electrons can be accommodated at lower energy. This downward shift of the band bottom may overcome the increase of the Fermi level due to the increased $n$ and an overall decrease of $\mu$ may occur, leading to a negative compressibility. 
Filling the bands in Eqs. (112) one easily gets the general expression of $\mu$ as a function of $n=n_{0}+\delta n$ at a fixed gate potential $V_{g}$. At lower densities, when only the isotropic band is involved, one obtains

$$
\mu\left(n, V_{g}\right)= \begin{cases}\frac{n^{2}}{\left(2 N_{0}\right)^{2} \varepsilon_{0}\left(n, V_{g}\right)}-\varepsilon_{0}\left(n, V_{g}\right) & \mu<0 \\ \frac{n}{N_{0}}-2 \varepsilon_{0}\left(n, V_{g}\right) & \mu \geq 0 .\end{cases}
$$

Experiments are usually performed at a fixed $V_{g}$ (and therefore at fixed $\delta n$ ) and a stable system uniformly reconstructs the surface forming a uniform $n_{0}$ electron density at the interface to avoid the polarity catastrophe. We now ask whether the system could be unstable and, at fixed $V_{g}$, could display a tendency to inhomogeneous reconstructions and a negative compressibility upon varying $n$ (or, equivalently, $n_{0}$ since $\delta n$ is kept fixed).

The inverse compressibility $\kappa^{-1}=\partial \mu / \partial n$ at fixed $V_{g}$ for the isotropic band reads

$$
\kappa^{-1}= \begin{cases}\frac{n}{2 N_{0}^{2} \varepsilon_{0}}-\frac{\partial \varepsilon_{0}}{\partial n}\left[\left(\frac{n}{2 N_{0} \varepsilon_{0}}\right)^{2}+1\right] & \mu<0 \\ \frac{1}{N_{0}}-2 \frac{\partial \varepsilon_{0}}{\partial n} & \mu \geq 0 .\end{cases}
$$

and can be negative in both the low-density $(\mu<0)$ and in the high-density $(\mu \geq 0)$ regime. The condition $\kappa<0$ naturally implies an electronic phase separation, i.e. a separation of the system into regions of different electronic densities to be determined by the standard Maxwell construction on the $\mu$ vs. $n$ curve.

An analysis of Eq. (4) for $\mu<0$ shows that if the electric field (and therefore $\alpha$ ) stays finite when $n=n_{0}+$ $\delta n \rightarrow 0$ (i.e. $E(0) \neq 0$ ), then $\kappa^{-1} \rightarrow-\partial \varepsilon_{0} / \partial n<0$ and the system is always driven unstable at sufficiently low filling. Although, depending on the value of $\alpha$, this latter instability could occur at very low densities [the $\delta n=-0.02$ curve (red online) in Fig. 2(b) displays a barely visible negative initial slope around $n_{0}=0.02$ (i.e. $n \approx 0)$ ], this observation might turn out to be relevant in physical systems like, e.g., MOSFETs or semiconducting heterostructures (see, e.g., Ref. 33]) where $\alpha$ is small but $n$ also is very small. In this regard, we considered the possibility that RSOC mechanism could be at the origin of the negative compressibility recently observed in LAO/STO interfaces [13] at low filling. We find that a negative compressibility in the observed range $n \sim 10^{12}$ $\mathrm{cm}^{-2}$ would require values of $\alpha \sim 5 \times 10^{-11} \mathrm{eVm}$.

On the other hand, in the case $\mu \geq 0$ of Eq. (4) we can take the limit of not too large fields $\beta E \ll 1$ [and $\left.E \approx n E^{\prime}\right]$ and obtain $\kappa^{-1} \approx n^{-1}\left(n / N_{0}-4 \varepsilon_{0}\right)$ which gives the simple condition $n / N_{0}<4 \varepsilon_{0}$ for the negative compressibility to occur. For instance, at densities $n \sim$ $10^{13} \mathrm{~cm}^{-2}, \kappa<0$ would require values as large as $\alpha \sim$ $6 \times 10^{-11} \mathrm{eV} \mathrm{m}$. It is also important to notice that a large
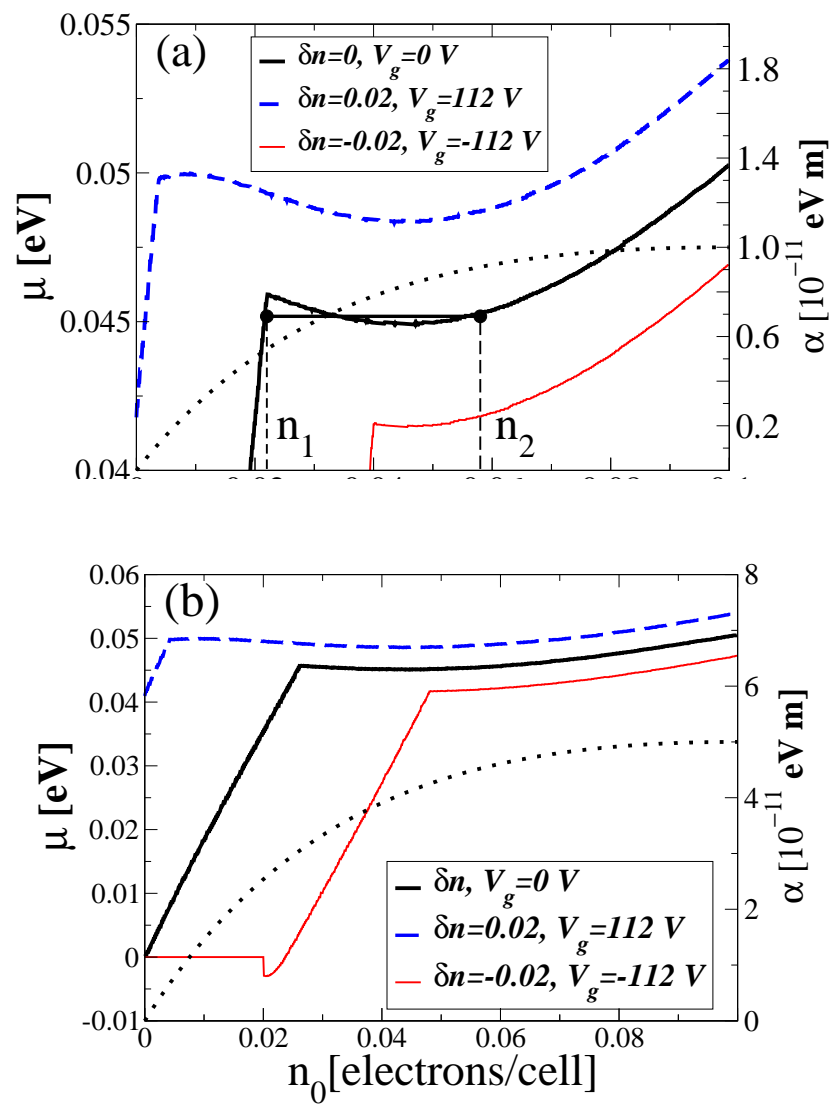

FIG. 2. (Color online) Chemical potential as a function of reconstructed electron density $n_{0}$ [0.01 electrons/cell correspond to $\left.6,25 \times 10^{12} \mathrm{~cm}^{-2}\right]$ for the three $t_{2 g}$ bands of STO. The lowest band is isotropic $(\nu=\eta=1)$ with mass $m \sim 0.7 m_{0}$. The two bands lying $\Delta=50 \mathrm{meV}$ above are anisotropic with $\nu=30$ and $\eta=1$ (a) or $\eta=1 / \nu$ (b). The legenda report the values of the additional electrons induced by gating. In (a) a Maxwell construction is also reported for the $V_{g}=0$ case. The dotted curves in (a) and (b) (right axes) are $\alpha$ as a function of $n=n_{0}\left(V_{g}=0\right)$.

DOS $N_{0}$ decreases the positive contribution to $\kappa^{-1}$ and favors the occurrence of the instability.

Recent magneto-conductivity experiments in LAO/STO interfaces find substantial values for $\alpha \sim 10^{-12} \div 10^{-11} \mathrm{eVm}$ 17, 18]. Similar values have been obtained for LTO/STO interfaces [35]. These values are about $5-8$ times smaller than those estimated above to drive the system unstable. In the specific case of very small densities [13], this quantitative discrepancy might well be due to the lack of many-body effects and disorder in our schematic model [34]). The question then arises whether at higher densities $n \sim 2 \div 4 \times 10^{13} \mathrm{~cm}^{-2}$, with the anisotropic bands partially filled, LXO/STO interfaces displays a negative compressibility at lower values of $\alpha$. This question is addressed by Fig. 2, which is the main outcome of our analysis and demonstrates the possibility of an inhomogenous electronic reconstruction as soon as the anisotropic bands begin to fill. While 
the $\eta=1 / \nu$ case $[$ panel (b)] only becomes unstable for large values of $\alpha \sim 1 \div 5 \times 10^{-11} \mathrm{eVm}$, the $\eta=1$ case [panel (a)] displays electronic phase separation for quite realistic values of $\alpha \sim 0.1 \div 1 \times 10^{-11} \mathrm{eVm}$, well within the range of those found in Refs. 17 and 18 in $\mathrm{LAO} / \mathrm{STO}$.

To provide a clear understanding of this result, we consider the following expressions

$$
\begin{aligned}
& n=2 N_{0} \varepsilon_{0}[1+2 f(\nu, \eta)]+\mu N_{0}+2(\mu-\Delta) N_{0} \sqrt{\nu}, \\
& \mu=\frac{1}{1+2 \sqrt{\nu}}\left\{\frac{n}{N_{0}}-2 \varepsilon_{0}[1+2 f(\nu, \eta)]+2 \Delta \sqrt{\nu}\right\}
\end{aligned}
$$

valid for $\mu>\Delta$ and $n>2 N_{0} \varepsilon_{0}[1+2 f(\nu, \eta)]+\Delta N_{0}$, respectively. Here, $f(\nu, \eta)$ is a (rapidly) increasing function of the mass anisotropy $\nu$ (see Supplemental Material) and gives a moderate $[f(30,1 / 30) \sim 3]$ to strong $[f(30,1) \sim 90]$ contribution to the negative term in Eq. (6). This shows that the RSOC-mediated instability takes advantage from the mass anisotropy and a negative compressibility is now more likely to occur.

For $\mu<\Delta$ the linear growth of $\mu$ in Fig. 2 shows that the DOS of the isotropic $d_{x y}$ band is too small to promote an instability for the values of $\alpha(n)$ given by the dot-dashed curves. On the other hand, as soon as the density $n_{0}+\delta n$ is large enough, the chemical potential eventually enters the anisotropic bands, the DOS rapidly increases (see, e.g., Ref. 29]) and $\mu$ decreases with $n_{0}$ signaling a negative compressibility region. For instance, the $V_{g}=0$ curve in Fig. 2(a) has negative slope in the range $n_{0} \sim 0.02-0.04$ electrons/cell $\left(\sim 3-5 \times 10^{13}\right.$ $\mathrm{cm}^{-2}$ ), which are typical values of as-grown systems. Even for lower values of $n_{0}$, the instability eventually occurs upon increasing the density with $V_{g}$ (see, e.g., the $V_{g}=112 \mathrm{~V}$ (blue online) dashed curve). This leads to a thermodynamic phase separation into regions of different densities (e.g., $n_{1}=0.022$ and $n_{2}=0.058$ in the $V_{g}=0$ curve of Fig. 2(a)) determined by a standard Maxwell construction [36]. For any given filling $n$, with $n_{1}<n<n_{2}$, the Maxwell construction also determines the relative weight of the two phases at $n_{1}$ and $n_{2}$.

A few remarks are now in order. Firstly, we point out that the band structure of the 2DEG at the LXO/STO interfaces is substantially modified by the RSOC arising from the strong electric field due to the polarity catastrophe. Therefore, it is not surprising that angle-resolved photoemission experiments 24 26] on STO/vacuum interfaces do not show the strong band splitting that one would expect from the experiments of Refs. [17, 18].

Secondly, we stress that additional mechanisms are also present in the real systems, which may cooperate with the intrinsic RSOC mechanism to produce inhomogeneous charge distributions. Indeed, if the electronic system has a large (although still positive) compressibility, impurities and defects more easily induce large inhomogeneities in such a "soft" and largely fluctuat- ing electron gas. In other words, they act as "external fields" on the density and may enhance the effects of the RSOC to locally induce phase separation. Previous work in strongly correlated systems [21, 37-41], also shows that electron-electron correlations and electron-phonon coupling favor phase separation. In this regard, the recent analysis of RSOC within Hartree-Fock approximation [42], can be a starting point for investigating the role of interactions on the RSOC-mediated instability.

Finally, we mention that our schematization of the LXO/STO interface in terms of a 2DEG should naturally be relaxed to account for the finite width of the interfacial electron gas [23 27]. However, the introduction of subbands due to this finite width will surely increase the DOS and consequently decrease the $\mu$ vs. $n$ slope when the chemical potential progressively crosses the subbands. This will strongly favor the instability further reducing the RSOC required for it.

In conclusion, within our rather essential description of the LXO/STO interface, we find that electronic phase separation may likely occur at oxide interfaces, where substantial density-dependent electric fields can arise. We stress that a negative compressibility (cf. Ref. [13]) cannot be explained by impurities, defects and so on, but is a distinct signature of an intrinsic mechanism leading to an effective charge segregating attraction like the one proposed here. We also show that (anisotropic) large masses (and subband splitting), isotropic RSOC, and low fillings favor the instability. This provides a natural framework accounting for the inhomogeneous phases observed in LXO/STO interfaces. It would be interesting to investigate the possible occurrence of electronic phase separation in cases where the RSOC is sizable and/or the density is very low, like in quantum wells, in boundaries of heavy metal alloys like $\mathrm{Bi}_{x} \mathrm{~Pb}_{1-x}$, in reconstructed surfaces of $\mathrm{Ag}(111)$ [43, 44], in MOSFET and semiconducting heterostructures at low densities, and in surface states of topological insulators [32, 45].

We thank N. Bergeal, J. Biscaras, V. Brosco, C. Castellani, C. Di Castro, T. Kopp, J. Lesueur, and R. Raimondi for stimulating discussions and J. Mannhart for bringing Ref. 13 to our attention. We acknowledge financial support from "University Research Project" of the "Sapienza" University n. C26A115HTN.

[1] A Ohtomo and H. Y. Hwang, Nature (London) 427, 423 (2004)

[2] N. Reyren, S. Thiel, A. D. Caviglia, L. Fitting Kourkoutis, G. Hammerl, C. Richter, C. W. Schneider, T. Kopp, A.-S. Retschi, D. Jaccard, M. Gabay, D. A. Muller, J.-M. Triscone, and J. Mannhart, Science 317, 1196 (2007).

[3] D. Caviglia, S. Gariglio, N. Reyren, D. Jaccard, T. Schneider, M. Gabay, S. Thiel, G. Hammerl, J. 
Mannhart, and J.-M. Triscone, Nature (London) 456 , 624 (2008).

[4] J. Biscaras, N. Bergeal, A. Kushwaha, T. Wolf, A. Rastogi, R. C. Budhani, and J. Lesueur, Nat. Commun., DOI: 10.1038 , and arXiv:1002.3737

[5] J. Biscaras, N. Bergeal, S. Hurand, C. Grossetête, A. Rastogi, R. C. Budhani, D. LeBoeuf, C. Proust, J. Lesueur, Phys. Rev. Lett. 108, 247004 (2012).

[6] S. Caprara, M. Grilli, L. Benfatto, and C. Castellani, Phys. Rev. B 84, 014514 (2011).

[7] D. Bucheli, S. Caprara, C. Castellani, and M. Grilli, arXiv:1205:0454

[8] Ariando, X. Wang, G. Baskaran, Z. Q. Liu, J. Huijben, J. B. Yi, A. Annadi, A. Roy Barman, A. Rusydi, S. Dhar, Y. P. Feng, J. Ding, H. Hilgenkamp, and T. Venkatesan, Nat. Commun., DOI: 10.1038/ncomms1192.

[9] J. A. Bert, B. Kalisky, C. Bell, M. Kim, Y. Hikita, H. Y. Hwang, and K. A. Moler, Nat. Phys. 7, 767 (2011).

[10] Lu Li, C. Richter, J. Mannhart, and R. C. Ashoori, Nat. Phys. 7, 762 (2011)

[11] Z. Ristic, R. Di Capua, G. M. De Luca, F. Chiarella, G. Ghiringhelli, J. C. Cezar, N. B. Brookes, C. Richter, J. Mannhart and M. Salluzzo, EPL 93, 17004 (2011) .

[12] N. C. Bristowe, T. Fix, M. G. Blamire, P. B. Littlewood, and Emilio Artacho, Phys. Rev. Lett. 108, 166802 (2012).

[13] Lu Li, C. Richter, S. Paetel, T. Kopp, J. Mannhart, R. C. Ashoori, Science 332, 825 (2011)

[14] N. Nakagawa, H. Y. Hwang, and D. A. Muller, Nat. Mat. 5, 204 (2006)

[15] Z. S. Popović, S. Satpathy, and R. M. Martin, Phys. Rev. Lett. 101, 256801 (2008)

[16] While Ref. 14 proposed a full electronic reconstruction with a large transfer of electrons at the interface of about 0.5 electron per cell, both experiments and more realistic calculations find much lower densities. See, e.g., M. Hirayama and M. Imada, J. Phys. Soc. Jpn. 79, 034704 (2010).

[17] A. D. Caviglia, M. Gabay, S. Gariglio, N. Reyren, C. Cancellieri, and J.-M. Triscone, Phys. Rev. Lett. 104, 126803 (2010).

[18] A. Fête, S. Gariglio, A. D. Caviglia, J.-M. Triscone, and M. Gabay, arXiv:1203.5239.

[19] V. J. Emery and S. Kivelson, Physica C 209, 597 (1993).

[20] R. Raimondi, C. Castellani, M. Grilli, Yunkyu Bang, and G. Kotliar, Phys. Rev. B 47, 3331 (1993); J. Lorenzana, C. Castellani, and C. Di Castro, Phys. Rev. B 64, 235127 (2001); R. Jamei, S. Kivelson, and B. Spivak, Phys. Rev. Lett. 94, 056805, (2005); C. Ortix, J. Lorenzana, C. Di Castro, Phys. Rev. Lett. 100, 246402 (2008).

[21] C. Castellani, C. Di Castro and M. Grilli, Phys. Rev. Lett. 75, 4650 (1995).

[22] L. F. Mattheiss, Phys. Rev. B 6, 4718 (1972).

[23] P. Delugas, A. Filippetti, V. Fiorentini, D. I. Bilc, D. Fontaine, and P. Ghosez, Phys. Rev. Lett. 106, 166807 (2011)

[24] A. F. Santander-Syro, O. Copie, T. Kondo, F. Fortuna, S. Pailhès, R. Weht, X. G. Qiu, F. Bertran, A. Nicolaou, A. Taleb-Ibrahimi, P. Le Fèvre, G. Herranz, M. Bibes, N. Reyren, Y. Apertet, P. Lecoeur, A. Barthélémy, and M. J. Rozenberg, Nature 469, 189 (2011).

[25] W. Meevasana, P. D. C. King, R. H. He, S-K. Mo, M. Hashimoto, A. Tamai, P. Songsiriritthigul, F. Baumberger, and Z-X. Shen, Nat. Mat. 10, 114 (2011).
[26] P. D. C. King, R. H. He, T. Eknapakul, P. Buaphet, S.-K. Mo, Y. Kaneko, S. Harashima, Y. Hikita, M. S. Bahramy, C. Bell, Z. Hussain, Y. Tokura, Z.-X. Shen, H. Y. Hwang, F. Baumberger, and W. Meevasana, Phys. Rev. Lett. 108, 117602 (2012).

[27] M. Salluzzo, J. C. Cezar, N. B. Brookes, V. Bisogni, G. M. De Luca, C. Richter, S. Thiel, J. Mannhart, M. Huijben, A. Brinkman, G. Rijnders, and G. Ghiringhelli, Phys. Rev. Lett. 102, 166804 (2009).

[28] This mixing was recently claimed to play a role in transport [29], but it provides only minor quantitative corrections to our results. For simplicity we neglect it.

[29] Arjun Joshua, S. Pecker, J. Ruhman, E. Altman and S. Ilani, arXiv:1110.2184.

[30] R. Winkler, Spin-Orbit Coupling Effects in TwoDimensional Electron and Hole Systems, SpringerVerlag, Berlin, Heidelberg, New York.

[31] Th. Schäpers, G. Engels, J. Lange, Th. Klocke, M. Hollfelder, and H. Lüth, J. Appl. Phys., 83, 4324 (1998).

[32] P. D. C. King, R. C. Hatch, M. Bianchi, R. Ovsyannikov, C. Lupulescu, G. Landolt, B. Slomski, J. H. Dil, D. Guan, J. L. Mi, E. D. L. Rienks, J. Fink, A. Lindblad, S. Svensson, S. Bao, G. Balakrishnan, B. B. Iversen, J. Osterwalder, W. Eberhardt, F. Baumberger, and Ph. Hofmann, Phys. Rev. Lett. 107, 096802 (2011).

[33] S. Ilani, A. Yacoby, D. Mahalu, Hadas Shtrikman, Science 292, 1354 (2001).

[34] Several important differences make a precise comparison with the experimental data of Ref. 13 difficult. First of all the top-gating used in experiments act on the filling differently from the back-gating of our model in Fig. 1(a). Secondly the experimental inverse compressibility values greatly depend on the samples, making a quantitative comparison hard to implement. Finally, some physical effects like electron-electron interaction and disorder become more important at low density, thereby preventing a reliable quantitative use of our schematic model.

[35] J. Biscaras, N. Bergeal, J. Lesueur, private communication.

[36] The Maxwell construction only determines the densities of the coexisting phases, but says nothing on the size and structure of the phase separated regions. The separation in differently charged regions may occur without paying a (large) Coulombic energy cost because the phase separated interface is inserted between the conducting leeds of the gating potential and, more importantly, because the polarity catastrophe and the related electronic reconstruction mechanism provide a suitable amount of countercharges, which can be locally rearranged to reestablish a long-distance charge neutrality. The possible formation of nano-, micro-, or meso-scopic clusters depends on several factors like the energy cost of cluster interfaces, screening length of the Coulomb electron-electron repulsion, distance from the countercharges at the surface of the top layer. Examples of Coulomb-frustrated phase separation are considered in Refs. 20.

[37] M. Grilli and C. Castellani, Phys. Rev. B 50, 16880 (1994).

[38] M. Grilli, R. Raimondi, C. Castellani, C. Di Castro, and G. Kotliar, Int. J. Mod. Phys. B 5, 309 (1991); Phys. Rev. Lett. 67, 259 (1991).

[39] F. Becca, M. Tarquini, M. Grilli, and C. Di Castro, Phys. Rev. B 54, 12443 (1996).

[40] Y. Bang, G. Kotliar, C. Castellani, M. Grilli, and R. 
Raimondi, Phys. Rev. B 43, 13724 (1991).

[41] N. Cancrini, S. Caprara, C. Castellani, C. Di Castro, M. Grilli and R. Raimondi, Europhys. Lett. 14, 597 (1991).

[42] A. Agarwal, Stefano Chesi, T. Jungwirth, Jairo Sinova, G. Vignale, and Marco Polini, Phys. Rev. B 83, 115135 (2011).

[43] Christian R. Ast, Jürgen Henk, Arthur Ernst, Luca Moreschini, Mihaela C. Falub, Daniela Pacilé, Patrick
Bruno, Klaus Kern, and Marco Grioni, Phys. Rev. Lett. 98, 186807 (2007).

[44] Fabian Meier, Vladimir Petrov, Sebastian Guerrero, Christopher Mudry, Luc Patthey, Jürg Osterwalder, and J. Hugo Dil, Phys. Rev. B 79, 241408(R) (2009).

[45] H. Beidenkopf, P. Roushan, J. Seo, L. Gorman, I. Drozdov, Y. S. Hor, R. J. Cava, and A. Yazdani, Nat. Phys. 7, 939 (2011). 